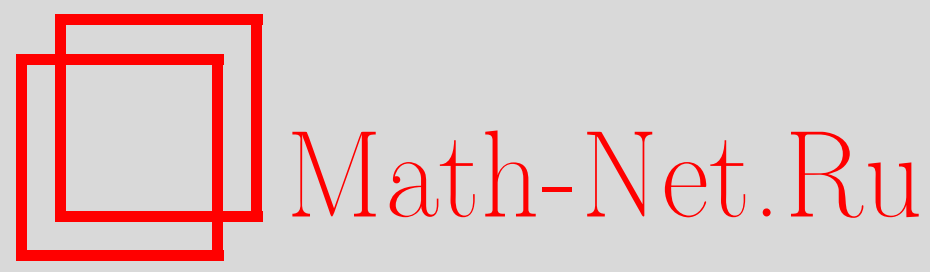

Г. И. Ивченко, Сколько потребуется выборок, чтобы увидеть все шары в урне?, Матем. заметки, 1998, том 64, выпуск $1,58-63$

DOI: https://doi.org/10.4213/mzm1372

Использование Общероссийского математического портала Math-Net.Ru подразумевает, что вы прочитали и согласны с пользовательским соглашением http://www.mathnet.ru/rus/agreement

Параметры загрузки:

IP: 3.80 .181 .102

26 апреля 2023 г., 13:18:39

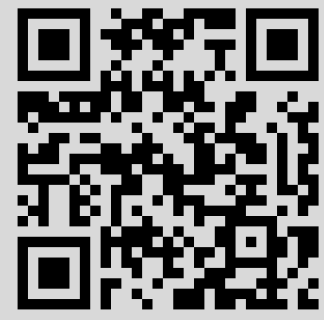




\section{СКОЛЬКО ПОТРЕБУЕТСЯ ВЫБОРОК, ЧТОБЫ УВИДЕТЬ ВСЕ ШАРЫ В УРНЕ?}

\section{Г.И. Ивченко}

Пусть урна содержит $N$ шаров, занумерованных числами от 1 до $N$. Из урны по схеме бесповторной выборки извлекается случайное число шаров, их номера запоминаются и шары возвращаются в урну. Эта процедура повторяется до тех пор, пока не будут зафиксированы в выборках все $N$ шаров. В предположении, что объемы последовательных выборок независимы и одинаково распределены, выводится аппроксимация для среднего числа необходимых выборок, а также его асимптотическое (при $N \rightarrow \infty$ ) распределение.

Библиография: 10 названий.

1. Введение. В литературе по случайньм размещениям частиц по ячейкам одной из популярных схем является схема размещения комплектами, упоминаемая еще в книге А. А. Маркова [1]. Классический вариант этой схемы формулируется следующим образом. В $N$ ячеек последовательно и независимо друг от друга бросаются комплекты по $m$ частиц, причем частицы каждого комплекта распределяются по разным ячейкам и все $\left(\begin{array}{l}N \\ m\end{array}\right)$ возможных размещений комплекта считаются равновероятньми. Исследованию различных характеристик такого процесса размещения частиц и его обобщений посвящена весьма обширная литература, отраженная, в частности, в монографиях [2], [3] и обзоре [4].

Одной из наиболее интересных характеристик этого процесса является число комплектов $\nu(N, m)$ (или время ожидания), после размещения которых впервые все ячейки оказьваются занятьми. Эта характеристика в точной и асимптотической $($ пи $N \rightarrow \infty)$ постановках достаточно детально исследована в работах Д. Пойа [5], А. Бекеши [6] и Г. И. Ивченко и Ю.И. Медведева [7]. В [8] предложен более общий вариант схемы, когда размеры комплектов являются случайньпи и представляют собой независимые копии целочисленной случайной величины $K$ с некоторым распределением $p_{m}=\mathrm{P}(K=m)$, $m=1, \ldots, N$. Для этого варианта время ожидания до заполнения всех ячеек обозначается через $\nu(N, K)$. Наше возвращение к этой теме стимулировано появлением работы T. Селке [9], в которой предложен оригинальный метод получения достаточно простой и одновременно весьма точной (что подтверждается численными расчетами) аппроксимации для $\mathrm{E} \nu(N, K)$ :

$$
\mathrm{E} \nu(N, K) \approx \sum_{i=0}^{N-1} \frac{1}{N-i} /\left(\sum_{i=0}^{N-1} \frac{1}{N-i} \mathrm{P}(K>i)\right)
$$

Работа выполнена при финансовой поддержке Российского фонда фундаментальных исследований, грант № 97-01-00387. 


$$
+\sum_{i=1}^{N-1} \frac{1}{N-i} \mathrm{P}(K>i) \sum_{j=1}^{i} \frac{1}{N-j+1} /\left(\sum_{i=0}^{N-1} \frac{1}{N-i} \mathrm{P}(K>i)\right)^{2} .
$$

Однако, предложенньй в [9] метод является довольно сложным и в то же время, так сказать, узко направленным, приспособленным именно для вычисления среднего $\mathrm{E} \nu(N, K)$. В данной заметке мы предлагаем простой альтернативный метод вычисления как точного значения $\mathrm{E} \nu(N, K)$, так и его последовательных аппроксимаций, которые одновременно являются асимптотическими разложениями среднего при больших $N$. Наш метод использует лиш прямые вероятностные и комбинаторные аргументы и позволяет также получить асимптотическое распределение времени ожидания $\nu(N, K)$ при $N \rightarrow \infty$, а также других характеристик схемы. Наш вариант аппроксимации имеет вид

$$
\mathrm{E} \nu(N, K) \approx \frac{N}{a_{1}} L_{N}+\frac{a_{1}-a_{2}}{2 a_{1}^{2}}\left(L_{N}-1\right), \quad L_{N}=\sum_{n=1}^{N} \frac{1}{n}, \quad a_{j}=\mathrm{E} K^{j}, j=1,2, \ldots ;
$$

при этом ее погрешность при больших $N$ имеет порядок $O((\ln N) / N)$.

В заключение отметим, что рассматриваемая задача может быть сформулирована также на язьке извлечения шаров из урны. Именно, пусть имеется урна, содержащая шары, занумерованные числами $1,2, \ldots, N$. Из урны по схеме бесповторной выборки извлекается случайное число шаров, их номера фиксируются и шары возврашаются в урну. Эта процедура повторяется до тех пор, пока не будут зафиксированы все $N$ шаров; при этом предполагается, что объемы последовательных выборок независимы и одинаково распределены как случайная величина $K$. Спрашивается: сколько для этого потребуется произвести выборок? Это и есть случайная величина $\nu(N, K)$.

2. Среднее значение времени ожидания. Мы исходим из известного представления математического ожидания целочисленной случайной величины через "хвосты" ее распределения:

$$
\mathrm{E} \nu(N, K)=\sum_{n=0}^{\infty} \mathrm{P}(\nu(N, K)>n) .
$$

В свою очередь, $\mathrm{P}(\nu(N, K)>n)=\mathrm{P}(\mu(N, n)>0)$, где $\mu(N, n)$ обозначает число пустых ячеек после размещения $n$ комплектов. Но

$$
\mu(N, n)=X_{1}+\cdots+X_{N}, \quad X_{i}= \begin{cases}1, & \text { если } i \text {-я ячейка пуста, } \\ 0 & \text { в противном случае }\end{cases}
$$

и по формуле для объединения событий

$$
\mathrm{P}(\mu(N, n)>0)=\mathrm{P}\left(\bigcup_{i=1}^{N}\left\{X_{i}=1\right\}\right)=\sum_{r=1}^{N}(-1)^{r-1} S_{r}
$$

где

$$
S_{r}=\sum_{1 \leqslant i_{1}<\cdots<i_{r} \leqslant N} \mathrm{P}\left(X_{i_{1}}=\cdots=X_{i_{r}}=1\right) .
$$

В данном случае суммы $S_{r}$ подсчитать не трудно. Именно, в силу симметрии и из определения схемы непосредственно следует, что

$$
S_{r}=\left(\begin{array}{c}
N \\
r
\end{array}\right) \mathrm{P}\left(X_{1}=\cdots=X_{r}=1\right)=\left(\begin{array}{c}
N \\
r
\end{array}\right)\left(\sum_{m=1}^{N} p_{m}\left(\begin{array}{c}
N-r \\
m
\end{array}\right) /\left(\begin{array}{l}
N \\
m
\end{array}\right)\right)^{n} .
$$


Преобразуем сумму, входящую в последнее выражение, к более удобному виду. Для этого, прежде всего, заметим, что

$$
\begin{aligned}
\left(\begin{array}{c}
N-r \\
m
\end{array}\right) /\left(\begin{array}{l}
N \\
m
\end{array}\right) & =\frac{(N-m)(N-m-1) \cdots(N-m-r+1)}{N(N-1) \cdots(N-r+1)}=\prod_{j=0}^{r-1}\left(1-\frac{m}{N-j}\right) \\
& =1-\sum_{s=1}^{r}(-1)^{s-1} m^{s} C_{s}(N, r),
\end{aligned}
$$

где

$$
C_{s}(N, r)=\sum_{0 \leqslant j_{1}<\cdots<j_{s} \leqslant r-1} \frac{1}{\left(N-j_{1}\right) \cdots\left(N-j_{s}\right)}
$$

Следовательно,

$$
\sum_{m=1}^{N} p_{m}\left(\begin{array}{c}
N-r \\
m
\end{array}\right) /\left(\begin{array}{l}
N \\
m
\end{array}\right)=1-\sum_{s=1}^{r}(-1)^{s-1} a_{s} C_{s}(N, r) \equiv 1-A_{r}(N)
$$

где $a_{s}=\mathrm{E} K^{s}, s=1,2, \ldots$ Таким образом,

$$
S_{r}=\left(\begin{array}{c}
N \\
r
\end{array}\right)\left(1-A_{r}(N)\right)^{n}
$$

и, тем самым,

$$
\mathrm{P}(\mu(N, n)>0)=\sum_{r=1}^{N}(-1)^{r-1}\left(\begin{array}{c}
N \\
r
\end{array}\right)\left(1-A_{r}(N)\right)^{n} .
$$

Отсюда и из (1) имеем следующее итоговое представление для среднего времени ожидания в нашей схеме:

$$
\mathrm{E} \nu(N, K)=\sum_{r=1}^{N}(-1)^{r-1}\left(\begin{array}{c}
N \\
r
\end{array}\right) A_{r}^{-1}(N)
$$

где $A_{r}(N)$ определено соотношениями (4) и (3).

При больших значениях $N$ вычисления по точной формуле (7) могут оказаться затруднительными, поэтому представляет практический (а не только теоретический) интерес получение простых и удобных аппроксимаций для $\mathrm{E} \nu(N, K)$. Идея построения таких аппроксимаций состоит в следующем.

Для величины $A_{r}^{-1}(N)$ при больших $N$ можно записать асимптотическое разложение

$$
A_{r}^{-1}(N)=\sum_{i \geqslant-1} \frac{b_{i}(r)}{N^{i}}
$$

где, как нетрудно установить, первые коэффициенты имеют вид

$$
\begin{gathered}
b_{-1}(r)=\frac{1}{r a_{1}}, \quad b_{0}(r)=\left(\frac{1}{r}-1\right) \frac{a_{1}-a_{2}}{2 a_{1}^{2}} \\
b_{1}(r)=\left(\frac{1}{r}-1\right)\left(r c_{1}+c_{0}\right) \frac{1}{12 a_{1}^{3}}, \quad c_{1}=a_{1}^{2}+2 a_{1} a_{3}-3 a_{2}, \quad c_{0}=a_{1}^{2}-4 a_{1} a_{3}+3 a_{2} .
\end{gathered}
$$


Подстановка этого разложения в (7) и использование известных комбинаторных тождеств

$$
\sum_{r=1}^{N}(-1)^{r-1}\left(\begin{array}{l}
N \\
r
\end{array}\right) r^{i}= \begin{cases}L_{N}=\sum_{n=1}^{N} \frac{1}{n}, & i=-1, \\
1, & i=0, \\
0, & 0<i<N,\end{cases}
$$

дает соответствующее асимптотическое разложение для $\mathrm{E} \nu(N, K)$. Учитьвая при этом лишь нескольких первых членов в (8), получаем соответствующую аппроксимацию, тем более точную, чем большее число членов разложения (8) используется. Для практических целей вполне достаточно приближения

$$
\mathrm{E} \nu(N, K) \approx \frac{N}{a_{1}} L_{N}+\frac{a_{1}-a_{2}}{2 a_{1}^{2}}\left(L_{N}-1\right),
$$

получаемого при учете лишь первых двух членов разложения (8). Если сделать еще один шаг, то мы получим дополнительное к правой части (10) слагаемое $\left(c_{0} L_{N}+c_{1}-c_{0}\right) /$ $\left(12 a_{1}^{3} N\right)$, которое уже убывает как $O((\ln N) / N)$ при больших $N$.

Отметим также, что апроксимация (10) согласуется с соответствующим результатом для схемы с фиксированньм размером комплектов $(K=m=$ const), который имеет вид [7]

$$
\mathrm{E} \nu(N, m)=\left(\frac{N}{m}-\frac{m-1}{2 m}\right) L_{N}+\frac{m-1}{2 m}+O\left(\frac{\ln N}{N}\right) .
$$

3. Асимптотические распределения. Разработанная выше техника позволяет также получить асимптотические (при $N \rightarrow \infty$ ) распределения случайных величин $\mu(N, n)$ и $\nu(N, K)$.

Рассмотрим сначала число $\mu(N, n)$ пустых ячеек после размещения $n$ комплектов. Из представления (2), как известно, следует, что его биномиальньй момент

$$
\gamma_{r}=\mathrm{E}\left(\begin{array}{c}
\mu(N, n) \\
r
\end{array}\right)
$$

равен $S_{r}$. Следовательно, в силу (5)

$$
\gamma_{r}=\left(\begin{array}{c}
N \\
r
\end{array}\right)\left(1-A_{r}(N)\right)^{n} .
$$

Но из (3) и (4) следует, что

$$
A_{r}(N)=\frac{r a_{1}}{N}\left(1+O\left(\frac{1}{N}\right)\right) \quad \text { при } N \rightarrow \infty,
$$

поэтому для любого $r=1,2, \ldots$

$$
\gamma_{r}=\frac{1}{r !}\left(N \exp \left\{-\frac{n a_{1}}{N}\left(1+O\left(\frac{1}{N}\right)\right)\right\}\right)^{r}\left(1+O\left(\frac{1}{N}\right)\right) .
$$

Если теперь $n=n(N)$ выбрано так, что

$$
N e^{-n a_{1} / N} \rightarrow \lambda, \quad 0<\lambda<\infty,
$$

то $\gamma_{r} \mapsto \lambda^{r} / r$ ! . Здесь предел есть $r$-й биномиальньй момент пуассоновой случайной величины с параметром $\lambda$. Тем самьм, распределение величины $\mu(N, n)$ при условии (11) сходится к распределению Пуассона П $(\lambda)$. Этому результату можно придать следующую форму. 
TEорема 1. Пусть $N \rightarrow \infty u n=N(\ln N+x+o(1)) / a_{1}$, әде $x-$ любое действительное число. Тогда $\mathscr{L}(\mu(N, n)) \rightarrow \Pi\left(e^{-x}\right)$.

Учитывая связь между распределениями $\nu(N, K)$ и $\mu(N, n)$, из этой теоремы непосредственно получаем также следующее утверждение.

Tеорема 2. Если $N \rightarrow \infty$, mo

$$
\mathrm{P}\left(\frac{a_{1}}{N} \nu(N, K)-\ln N \leqslant x\right) \rightarrow e^{-e^{-x}}, \quad-\infty<x<\infty .
$$

Аналоги теорем 1 и 2 для схемы с фиксированньм размером комплектов хорошо известны. Подчеркнем, что асимптотические распределения рассматриваемых характеристик в общем случае зависят лиш от среднего размера комплектов $a_{1}=\mathrm{E} K$ (при условии, что распределение случайной величины $K$ фиксировано и не зависит от $N$ ).

4. Обобщения. Возможности нашегометода не исчерпываются изложенным. Здесь мы кратко укажем некоторые его обобщения.

1) Рассмотрим сначала задачу вычисления дисперсии времени ожидания. Если воспользоваться известной формулой $[10$, гл. XI, §1]

$$
\mathrm{D} \nu(N, K)=2 \sum_{n=0}^{\infty} n \mathrm{P}(\nu(N, K)>n)+\mathrm{E} \nu(N, K)(1-\mathrm{E} \nu(N, K)),
$$

то аналогично п. 2 получим представление

$$
\mathrm{D} \nu(N, K)=2 \sum_{r=1}^{N}(-1)^{r-1}\left(\begin{array}{c}
N \\
r
\end{array}\right) A_{r}^{-2}(N)-\mathrm{E} \nu(N, K)(1+\mathrm{E} \nu(N, K)) .
$$

Воспользовавшись (9) и тождеством

$$
\sum_{r=1}^{N}(-1)^{r-1}\left(\begin{array}{c}
N \\
r
\end{array}\right) \frac{1}{r^{2}}=\sum_{n=1}^{N} \frac{1}{n} L_{n}=\frac{1}{2}\left(L_{N}^{2}+\sum_{n=1}^{N} \frac{1}{n^{2}}\right)
$$

и учитьвая (10), не трудно получить апшроксимацию

$$
\mathrm{D} \nu(N, K) \approx \frac{N^{2}}{a_{1}^{2}} \sum_{n=1}^{N} \frac{1}{n^{2}}-\frac{a_{1}^{2}+a_{1}-a_{2}}{a_{1}^{3}} N L_{N}+N \frac{a_{1}-a_{2}}{a_{1}^{3}} \sum_{n=1}^{N} \frac{1}{n^{2}} .
$$

В частности, для схемы с фиксированным размером $m$ комплектов это сводится к соотношению

$$
\mathrm{D} \nu(N, m) \approx \frac{N^{2}}{m^{2}} \sum_{n=1}^{N} \frac{1}{n^{2}}-\frac{N}{m^{2}} L_{N}-N \frac{m-1}{m^{2}} \sum_{n=1}^{N} \frac{1}{n^{2}},
$$

что согласуется с соответствующим результатом из [7].

2) Пусть $\nu_{t}(N, K)$ обозначает число комплектов, после размешения которых впервые окажутся занятьми не менее $t$ ячеек (так что $\nu_{N}(N, K)=\nu(N, K)$ ). Тогда, очевидно,

$$
\mathrm{P}\left(\nu_{t}(N, K)>n\right)=\mathrm{P}(\mu(N, n)>s), \quad s=N-t,
$$


поэтому вместо (6) здесь надо использовать формулу для вероятности осуществления по крайней мере $s+1$ событий $[10$, гл. IV,$\S 5]$ :

$$
\mathrm{P}(\mu(N, n)>s)=\sum_{r=1}^{N}(-1)^{r-1-s}\left(\begin{array}{c}
r-1 \\
s
\end{array}\right)\left(\begin{array}{c}
N \\
r
\end{array}\right)\left(1-A_{r}(N)\right)^{n} .
$$

В итоге для среднего времени ожидания мы получаем представление

$$
\mathrm{E} \nu_{t}(N, K)=\sum_{r=1}^{N}(-1)^{r-1-s}\left(\begin{array}{c}
r-1 \\
s
\end{array}\right)\left(\begin{array}{c}
N \\
r
\end{array}\right) A_{r}^{-1}(N),
$$

обобщающее (7). Если $s>0$, то

$$
\left(\begin{array}{c}
r-1 \\
s
\end{array}\right)=(-1)^{s} \prod_{j=1}^{s}\left(1-\frac{r}{j}\right)=(-1)^{s}\left(1-r L_{s}+\cdots\right),
$$

и по стандартной схеме вывода аппроксимации (10) мы получаем соотношение

$$
\mathrm{E} \nu_{t}(N, K) \approx \frac{N}{a_{1}}\left(L_{N}-L_{s}\right)+\frac{a_{1}-a_{2}}{2 a_{1}^{2}}\left(L_{N}-L_{s}-1\right) .
$$

3) Отметим, наконец, обобщение теоремы 2 для времени ожидания $\nu_{t}(N, K)$.

Теорема 3. Пусть $N, t \rightarrow \infty$ так, что $s=N-t$ фиксировано. Тогда предельная функиия распределения $F_{s}(x)$ нормированной случайной величины $a_{1} \nu_{t}(N, K) / N-$ $\ln N$ имеет вид

$$
F_{s}(x)=e^{-e^{-x}} \sum_{r=0}^{s} \frac{e^{-r x}}{r !}, \quad-\infty<x<\infty .
$$

\section{СПИСОК ЦИТИРОВАННОЙ ЛИТЕРАТУРЫ}

[1] Markoff A. A. Wahrscheinlichkeitsrechnung. Leipzig-Berlin, 1912.

[2] Колчин В.Ф., Севастьянов Б.А., Чистяков В.П. Случайные размещения. М.: Наука, 1976.

[3] Коваленко И. Н., Л евитская А. А., Савчук М. Н. Избранные задачи вероятностной комбинаторики. Киев: Наукова думка, 1986.

[4] Иванов В.А., Ивченко Г. И., Медведев Ю.И. Дискретные задачи в теории вероятностей // Итоги науки и техн. Теория вероятн. Матем. статистика. Теоретич. кибернетика. Т. 22. М.: ВИНИТИ, 1984. С. 3-60.

[5] Polya G. Eine Wahrscheinlichkeitsaufgabe in der Kundenwerbung // Z. Angew. Math. Mech. 1930. V. 10. № 1-3. P. 96-97.

[6] Bekessy A. A lottojatekal kapesolatos nehany cellabetoltesi problemazol. II // Mat. Lapok. 1965. V. 16. P. 57-67.

[7] Ивченко Г.И., Медведев Ю.И. Асимптотическое поведение числа комплектов частиц в классической задаче о размещении // Теория вероятн. и ее примен. 1966. Т. 11. № 4 . C. $701-708$.

[8] Ивченко Г. И. Некоторые предельные теоремы в схеме размещения // Некоторые прикладные вопросы теории вероятн. и матем. статистика. Тр. МИЭМ. Вьп. 32. М.: МИЭМ, 1973. C. 111-119.

[9] Sellke T. M. How many samples does it take to see all the balls in a box? // Ann. Appl. Probab. 1995. V. 5. №1. P. 294-309.

[10] Феллер В. Введение в теорию вероятностей и ее приложения. 2-е изд. М.: Мир, 1964. 